\title{
Controle Granular Fuzzy Evolutivo Aplicado à Navegação de Veículos Inteligentes
}

\author{
João Paulo de Oliveira Tavares* Danilo Alves de Lima** \\ Daniel Furtado Leite ${ }^{* * *}$

\begin{abstract}
* Departamento de Engenharia, Universidade Federal de Lavras, ** Departamento de Automática, Universidade Federal de Lavras, Lavras, Minas Gerais, Brasil (e-mail:danilo.delima@ufla.br)
\end{abstract} \\ Lavras, Minas Gerais, Brasil (e-mail: jp.tavares@posgrad.ufla.br). \\ *** Departamento de Automática, Universidade Federal de Lavras, \\ Lavras, Minas Gerais, Brasil (e-mail:daniel.leite@ufla.br)
}

\begin{abstract}
Resumo:
No últimos anos, diversos avanços em sistemas de condução inteligentes têm sido apresentados no mundo. Um dos desdobramentos de tais avanços é a utilização de veículos inteligentes a fim de trazer mais eficiência nos transportes, melhorar a mobilidade nos grandes centros, e aumentar a segurança dos condutores e pedestres em uma via. No entanto, diversos desafios de caráter técnico e não-técnico ainda devem ser vencidos para que os veículos possam atingir níveis de autonomia elevados e se tornarem populares nas ruas. Um desses desafios é garantir sua condução de forma segura e eficiente. Este trabalho tem como objetivo validar a utilização de uma abordagem de controle de navegação inteligente baseada em modelo fuzzy evolutivo de veículos inteligentes a partir do fluxo online de dados provenientes dos sensores embarcados no veículo. A modelagem e controle apresentados se mostraram satisfatórios para diversos casos.
\end{abstract}

\begin{abstract}
:
In recent years, several advances in intelligent driving systems have been presented worldwide. One of the consequences of such developments is the use of intelligent vehicles to make transport more efficient, improve mobility in major centers, and increase the safety of drivers and pedestrians. However, a number of technical and non-technical challenges are still to be addressed before vehicles can reach a high level of autonomy, and become popular on the streets. One of these challenges concerns with the assurance of safety, and an efficient navigation. This paper aims to validate the use of an intelligent navigation control approach based on evolving fuzzy model of intelligent vehicles from online data streams provided by embedded sensors. Encouraging results are achieved in several practical cases by the proposed evolving fuzzy model and controller.
\end{abstract}

Keywords: Intelligent Control; Evolving Systems; Granular Computing; Navigation; Intelligent Vehicles.

\section{INTRODUÇÃO}

Atualmente, os temas relacionados aos Sistemas Avançados de Assistência ao Condutor (ADAS) ${ }^{1}$ são alguns dos mais pesquisados no campo da indústria automotiva. A utilização de veículos inteligentes, ou seja, veículos equipados com algum sistema automatizado de condução, pode melhorar sensivelmente a segurança dos usuários, a eficiência dos transportes, a mobilidade da população, entre diversos outros aspectos (Singh, 2015). Devido a esse potencial, é uma área de estudos proeminente, sendo largamente pesquisada tanto pela academia, quanto pela indústria. A condução de forma segura e eficiente desses veículos depende, entre outras coisas, da utilização de bons sistemas de automação e controle.

\footnotetext{
1 Do inglês Advanced Driver Assistance Systems.
}

O grau de autonomia de um veículo inteligente foi internacionalmente estabelecido pela Sociedade dos Engenheiros Automotivos (SAE) em 6 níveis (SAE et al., 2016), dentre os quais os veículos de nível 3, 4 ou 5 são capazes de realizar seu controle de navegação com pouca ou sem a intervenção humana. Para que um veículo seja classificado entre os níveis mais elevados de autonomia, é fundamental modelar seus sistemas e controlá-lo com precisão e segurança. Neste contexto, a dinâmica envolvida na navegação de veículos inteligentes são incertas, não-lineares e não-estacionárias. Assim, a utilização de técnicas que sejam capazes de se ajustar de acordo com as diferentes circunstâncias mostrase vantajosa, uma vez que não há evidências que um sistema de controle projetado offline possa realizar suas funções adequadamente se o processo ou o ambiente em torno do sistema se altere (Landau et al., 2011).

Atualmente, os sistemas inteligentes evolutivos são um dos principais temas pesquisados na modelagem de da- 
dos online (Kasabov et al., 2017). Diversos trabalhos que envolvam sistemas evolutivos, ou seja, sistemas que sejam capazes de alterar e atualizar suas estruturas quando necessário a partir de um fluxo de dados, foram desenvolvidos nos últimos anos (Majdani et al., 2018) (Škrjanc et al., 2019). Este tipo de abordagem é adequada para lidar com situações em que não é possível prever algum acontecimento. Novas informações são integradas à um modelo global a partir de adaptação estrutural, enquanto modelos locais podem adaptar incrementalmente seus parâmetros a partir de informações descobertas no fluxo. Sistemas de controle inteligentes baseados em sistemas fuzzy são recomendados para lidar com problemas difíceis de formular e analisar a partir de equações diferenciais devido à parâmetros não-estacionários e incertezas (Cara et al., 2010). Um modelo fuzzy pode fornecer predições de valores futuros de variáveis e um conjunto de regras interpretáveis, combinando interpretabilidade e precisão provenientes, respectivamente, da parte linguística e funcional de consequentes de regras fuzzy (Abhishek et al., 2012). Dessa forma, é possível aproximar as saídas dos sistemas, e fornecer limites de tolerância em torno de uma previsão numérica (Leite et al., 2015).

Nesse sentido, o termo evolutivo pode ser tratado como o desenvolvimento gradual da base de regras fuzzy e os parâmetros dos conjuntos fuzzy e funções locais. Para isso, esse sistema deve ser capaz de aprender continuamente a partir do fluxo de dados; não armazenar amostras anteriores; não depender prioritariamente do conhecimento estrutural; auto-adaptar sua estrutura quando necessário; e ser independente da propriedade estatística dos dados (Škrjanc et al., 2019).

Este trabalho tem o objetivo de validar um controle de navegação de veículos inteligentes a partir de uma abordagem baseada no modelo granular fuzzy evolutivo, que é capaz de se auto-adaptar parametricamente e estruturalmente em tempo real. O trabalho foi organizado da seguinte forma: A Seção II mostra uma recapitulação de trabalhos que utilizam técnicas de inteligência computacional no controle de veículos autônomos. A Seção III apresenta o ambiente de modelagem e controle utilizado. Os resultados obtidos são mostrados na Seção IV. A Seção V mostra as conclusões do trabalho e as sugestões de trabalhos futuros.

\section{TRABALHOS RELACIONADOS}

Esta seção apresenta alguns estudos recentes sobre técnicas de inteligência computacional aplicadas ao controle de navegação de veículos inteligentes. A proposta é mostrar uma visão geral da literatura relacionada.

Um veículo inteligente é tradicionalmente subdividido em subsistemas de localização, navegação, percepção e controle (Campbell et al., 2010). Esses subsistemas possuem suas especificidades e funções delimitadas para realizar a tarefa de controlar a navegação do veículo. Com o advento das técnicas de inteligência computacional, existem aplicações que extrapolam essa estrutura tradicional de controle, trazendo mais eficiência e confiabilidade aos sistemas de condução autônomos.

Diversas técnicas de controle têm sido utilizadas para garantir que um veículo possa navegar com erros mínimos e com a maior segurança possível. Com o avanço gradativo das técnicas de inteligência computacional e de aprendizado de máquina, é cada vez mais comum a utilização de algoritmos fundamentados em conceitos e princípios destas teorias para controlar a navegação de veículos inteligentes. A finalidade muitas vezes é superar o desempenho alcançado com abordagens de controle convencionais. Nesse sentido, se destacam em termos de quantidade as aplicações que utilizam diferentes tipos de redes neurais para modelagem e processamento dos dados provenientes de sistemas autônomos.

São observadas na literatura diversas aplicações que envolvem redes neurais para tarefas como manter o veículo em uma via e desviar de obstáculos (Bojarski et al., 2016). A ideia é aprender a partir de dados obtidos a partir da condução do veículo por um humano (aprendizado por imitação). Nessas abordagens, uma entrada sensorial bruta, como uma imagem, sem pré-processamento, é utilizada para gerar comandos de controle automaticamente (Codevilla et al., 2017). Dessa forma, elimina-se a etapa intermediária de construção explícita de um modelo do ambiente. Diferentemente das abordagens tradicionais, no aprendizado por imitação, os dados coletados para a etapa de treinamento, podem ser obtidos com relativa facilidade, por exemplo, navegando um veículo e obtendo as imagens capturadas pelas câmeras com os respectivos valores dos ângulos de esterçamento do veículo.

Como na grande maioria das aplicações envolvendo redes neurais, o processo de decisão interno de um modelocontrolador neural é difícil de ser compreendido (estrutura dita caixa-preta). Isso faz surgir problemas relacionados à segurança uma vez que um sistema de direção não interpretável, ou incompreensível linguisticamente, é também pouco confiável (Sauer et al., 2018). Outra dificuldade do uso de modelos neurais diz respeito a necessidade de aprendizado offline a partir de bases de dados estáticas. Nesses casos, o controlador é incapaz de aprender recursivamente a partir do fluxo de dados online, obtido durante a navegação do veículo.

Em (Chen and Huang, 2017) é prevista uma representação que estipula diretamente as informações necessárias para controlar a navegação de um veículo. Evita-se a modelagem completa do ambiente entorno ou a captura de dados que reflitam o estado de todo o ambiente. Temse uma abordagem intermediária entre as representações tradicionais e o aprendizado por imitação direto, no qual o ambiente é representado por indicadores que descrevem sua situação, e.g., a distância às bordas da via, ao veículo à frente, entre outros. A partir dessa representação, o veículo é conduzido por meio de algum controlador capaz de proceder as decisões necessárias para a navegação.

Nesse contexto, utilizar uma abordagem que possua características evolutivas, pode ser potencialmente útil em um ambiente onde se é necessário grande robustez na tarefa de controle. Entre as principais vantagens, pode-se citar a independência de um modelo do veículo, pois a própria técnica é capaz de desenvolver e manter atualizado um modelo a partir do fluxo de dados online; e o baixo custo computacional atrelado à abordagem. $\mathrm{O}$ modelo evolutivo aprende sua estrutura e parâmetros incrementalmente a partir de novas situações ao qual o veículo se depara. 


\section{MODELAGEM E CONTROLE EVOLUTIVO GRANULAR}

Sistemas granulares evolutivos tratam-se de algoritmos incrementais que constroem a estrutura de modelos baseados em regras, e adaptam os termos antecedentes e consequentes dessas regras (Leite et al., 2015) (Angelov et al., 2010). Sistemas evolutivos geram novas regras sempre que as informações do fluxo de dados não condizem com o entendimento atual do processo (Leite et al., 2015). Nesse sentido, foi introduzido o conceito de controle granular fuzzy (Leite, 2012). Nesse tipo de abordagem, um modelo fuzzy é desenvolvido a partir de um fluxo de dados numéricos ou incertos, ditos dados granulares, e um algoritmo de aprendizado incremental. Os dados podem ser intervalos fuzzy, em geral, ao invés de valores numéricos pontuais, sujeitos a ruído e erros (Leite et al., 2015).

O controle granular evolutivo lida com informações numéricas ou fuzzy e fornece entradas de controle capazes de estabilizar um sistema. Informações em tempo real são úteis para evoluir o modelo do controlador para que seja possível atingir e manter um nível de performance desejado. Novos grânulos ou regras podem ser criados ou atualizados a partir de mudanças no fluxo de dados. Portanto, a abordagem de controle granular fuzzy é especialmente vantajosa quando o modelo do processo é desconhecido e/ou varia no tempo (Leite et al., 2015). Neste trabalho é utilizado um algoritmo de aprendizado de máquina incremental para gerar a estrutura e manter os parâmetros de um modelo fuzzy atualizados. Os atuadores de um veículo são acionados oportunamente, sem a intervenção humana. Os detalhes de tal ambiente de modelagem e controle são apresentados na seção a seguir.

\subsection{Modelagem Evolutiva Fuzzy}

Um modelo evolutivo é dito granular quando fundamentado em conceitos da teoria da Computação Granular. Modelos locais (grânulos de informação) são criados e desenvolvidos utilizando um algoritmo recursivo (Leite et al., 2012). O objetivo é fornecer informações úteis e soluções aproximadas para problemas do mundo real (Yao, 2010). Nesse contexto, um grânulo de informação define um subconjunto de um universo. Os responsáveis pela criação e adaptação dos grânulos de um modelo evolutivo são os dados de um fluxo. As amostras guiam a adaptação dos parâmetros associados ao modelo local (Leite et al., 2012). Além disso, modelos granulares facilitam a incorporação de conhecimento especialista e percepções do mundo físico. Assim, especialistas podem confrontar as informações do conjunto de regras que ditam a dinâmica do sistema, alterar seus parâmetros, e introduzir informações adicionais no modelo (Leite et al., 2015).

A criação de grânulos $\gamma^{i}, i=1, \ldots, c$, a partir de um fluxo de dados é ilustrada na Figura 1. Note que os dados são medições da função que descreve um processo. A projeção dos grânulos $\gamma^{i}$ nos eixos representando a variável de entrada, $x_{j}$ (em geral $\left.j=1, \ldots, n\right)$, e de saída, $y$, são funções de pertinência trapezoidais, denotadas por $A^{i} \mathrm{e}$ $B^{i}$, respectivamente.

Um controlador fuzzy evolutivo granular livre de modelo consiste em um conjunto de regras fuzzy no qual os termos
Figura 1. Obtenção de grânulos a partir de um conjunto de dados.

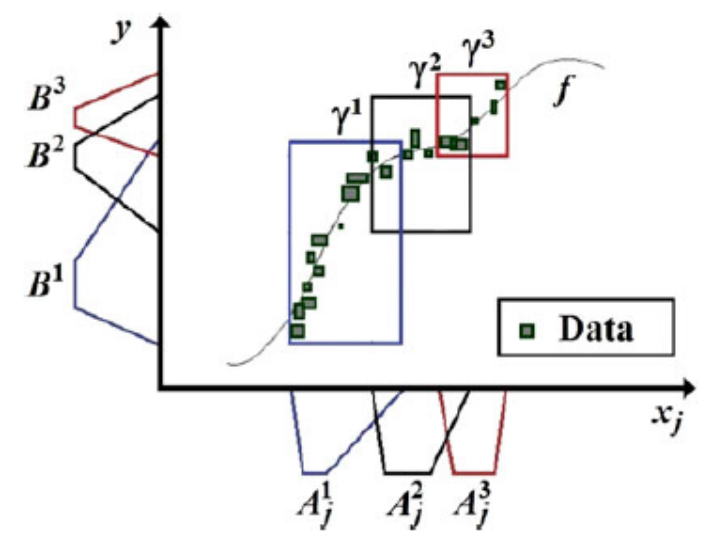

Fonte: (Leite et al., 2012)

antecedentes tratam-se de funções de pertinência. O termo consequente de uma regra é uma lei de controle com parâmetros variantes no tempo (Leite and Gomide, 2012). Dessa forma, o principal objetivo no projeto de um controlador desse tipo é encontrar cada regra do controlador em tempo real para estabilizar ou melhorar a dinâmica transitória das variáveis controladas. Em cada instante $k$, o sistema de controle fornece uma ação de controle $y(k)$ e, então, adapta o modelo-controlador. Assim, o modelocontrolador aprende e evolui seus parâmetros e estrutura online (Leite et al., 2015). Controladores granulares fuzzy evolutivos baseados em modelo são discutidos em (Leite et al., 2015). Nesse caso, garante-se estabilidade de Lyapunov do sistema em malha fechada a partir da factibilidade de inequações matriciais lineares (Boyd et al., 1994). $\mathrm{O}$ presente trabalho considera controle livre de modelo, conforme (Leite and Gomide, 2012).

Como as demais aplicações que se baseiam em conceitos fuzzy, um modelo granular fuzzy lida com as incertezas e imprecisões dos dados coletados (Bargiela and Pedrycz, 2008; Kasabov et al., 2017). O modelo considerado neste trabalho disponibiliza um conjunto de regras SEENTÃO criado e desenvolvido incrementalmente a partir da sequência de dados. Para cada grânulo de informação existe uma regra e uma descrição linguística corrrespondente. Em geral, não existem grânulos e regras prévias ao processo de aprendizagem (Leite et al., 2012). Assim, com a descoberta de novos padrões de comportamento, novos grânulos e regras são gerados, e os parâmetros do modelo fuzzy adaptados (Angelov et al., 2010) (Leite and Gomide, 2012). Em geral, um número finito de estados passados, $x(k), x(k-1), \ldots, x(k-m)$, entradas de controle $u(k), u(k-1), \ldots, u(k-n)$ e outras variáveis exógenas podem ser consideradas nos termos antecedentes das regras. Dessa forma, o conjunto de regras $R^{i}, i=1, \ldots, c$, que rege a operação do modelo-controlador tem a forma:

$$
\begin{array}{r}
R^{i}: \mathrm{SE}\left(x_{1}(k) \text { é } M_{1}^{i}\right) \mathrm{E} \ldots \mathrm{E}\left(x_{n}(k) \text { é } M_{n}^{i}\right) \\
\text { ENTÃO } y^{i}(k)=A^{i} x(k)
\end{array}
$$

Nesta expressão, $M_{j}^{i}=\left(l_{j}^{i}, \lambda_{j}^{i}, \Lambda_{j}^{i}, L_{j}^{i}\right)$ é a $i$-ésima função de pertinência trapezoidal definida no universo da variável 
$x_{j} . x(k)=\left[\begin{array}{lll}x_{1}(k) \ldots x_{n}(k)\end{array}\right]^{T}$ são os estados atuais do sistema, $y^{i}(k)$ é a ação de controle local. A ação de controle global, $y(k)$, é a média aritmética das ações (contribuições) locais ponderadas pelo grau de ativação da respectiva regra. O grau de ativação da $i$-ésima regra é dado pela T-norma, min, calculada para os graus de pertinência dos estados $x_{j}$ nos conjuntos fuzzy $M_{j}^{i}, j=1, \ldots, n$. Note que o número de regras $R^{i}, i=1, \ldots, c$, é variável (evolui) de acordo com a dinâmica do sistema.

Em geral, a matriz consequente $A^{i}$ de uma regra é retangular, depende do número de entradas e saídas, e possui termo livre, $a_{0}^{i}$. Para um modelo com $n$ entradas e uma saída, $A^{i} \in \Re^{1, n+1}$. Nesse caso, a ação de controle é uma combinação linear das entradas; uma função afim.

Uma função local busca aproximar a função desconhecida do sistema físico, $f$, no domínio de um grânulo de informação $\gamma^{i}$. A função local é adaptada por amostras de entrada pertencentes ao grânulo. Elas podem ser de diferentes tipos, e não necessariamente lineares. O algoritmo Mínimos Quadrados Recursivo (MQR) (Feng, 2002) é útil para calcular os coeficientes $a_{j}^{i}, j=0,1, \ldots, n$; Leite et al. (2012).

\subsection{Adaptação Online do Modelo}

A estrutura e os parâmetros do modelo fuzzy evolutivo são adaptados recursivamente a partir do fluxo de dados. Assim, os grânulos de informação e as regras fuzzy são atualizados à medida que o comportamento do processo muda. O mecanismo de aprendizado cria novos grânulos e regras na estrutura do modelo quando amostras de dados são muito diferentes do conhecimento atual. Caso um novo dado de entrada possa ser entendido a partir do conhecimento atual, os parâmetros dos grânulos já existentes podem ser adaptados. O processo de aprendizado se baseia, principalmente, na expansão e contração dos conjuntos fuzzy $M_{1}^{i}, \ldots, M_{n}^{i}$ de algumas regras $R^{i}$ para inclusão de novos dados. O algoritmo também atualiza os coeficientes das matrizes consequentes $A^{i}$ (Leite, 2012).

\subsection{Criação de Grânulos}

A estrutura adaptável do modelo se contrai ou expande a partir de uma região de expansão $E_{j}^{i}$ relativa ao conjunto fuzzy $M_{j}^{i}$. Um parâmetro, $\rho$, denominado granularidade máxima, define o quanto o suporte de um conjunto fuzzy trapezoidal pode alargar-se na tentativa de incluir uma amostra de dados. Caso uma amostra pertença à região de expansão de um grânulo, então ela pode ser incluída neste grânulo, alterando os parâmetros das funções de pertinência trapezoidais associadas. Caso a amostra não pertença à $E^{i}$, então é necessária a adição de um novo grânulo $\gamma^{c+1}$ ao conjunto atual, vide detalhes da abordagem em (Leite, 2012) (Leite and Gomide, 2012). Com a criação de um novo grânulo, uma nova regra $R^{c+1}$ é adicionada ao conjunto de regras $R=\left\{R^{1}, \ldots, R^{i}, \ldots, R^{c}\right\}$. Formalmente, a região de expansão $E_{j}^{i}$ é definida como:

$$
E_{j}^{i}=\left[m p\left(M_{j}^{i}\right)-\frac{\rho}{2}, m p\left(M_{j}^{i}\right)+\frac{\rho}{2}\right]
$$

em que $m p\left(M_{j}^{i}\right)$ é o ponto médio de $M_{j}^{i}$ obtido a partir da média dos parâmetros do núcleo do conjunto fuzzy tra- pezoidal, e $\rho$ representa a largura máxima que o conjunto em questão pode expandir. Portanto, se $x(k)$ estiver fora da região $E_{j}^{i}$ para algum $j, j=1, \ldots, n$, e para todos os grânulos $\gamma^{i}, i=1, \ldots, c$, então um novo grânulo será construído por meio de conjuntos $M_{j}^{c+1}, j=1, \ldots, n$ (Leite, 2012) conforme:

$$
\begin{array}{r}
M_{j}^{c+1}=\left(l_{j}^{c+1}, \lambda_{j}^{c+1}, \Lambda_{j}^{c+1}, L_{j}^{c+1}\right)=\left(x_{j}, x_{j}, x_{j}, x_{j}\right), \\
j=1, \ldots n .
\end{array}
$$

\subsection{Adaptação de Grânulos}

A adaptação de um grânulo $\gamma^{i}$ consiste em expandir ou contrair o suporte e o núcleo do termo antecedente das funções trapezoidais $M_{j}^{i}$ para acomodar novas informações e simultaneamente ajustar os coeficientes da matriz consequente $A^{i}$. Assim, uma regra deve ser adaptada sempre que uma observação estiver em uma região mapeada.

Dentre os grânulos $\gamma^{i}$ aptos a se expandirem para incluir uma amostra, aquele que tenha a maior similaridade, de acordo com a Equação 4, é escolhido.

$$
\begin{array}{r}
S\left(x_{j}, \gamma^{i}\right)=1-\frac{1}{6 n} \sum_{j=1}^{n}\left(2\left|\Lambda_{j}^{i}-x_{j}\right|+2\left|\lambda_{j}^{i}-x_{j}\right|+\right. \\
\left.+\left|l_{j}^{i}-x_{j}\right|+\left|L_{j}^{i}-x_{j}\right|\right) .
\end{array}
$$

Essa medida quantifica o grau que os dados de entrada combinam com o conhecimento local atual (Leite et al., 2012). Para o caso deste trabalho, a amostra $x_{j}$ é numérica. Logo, seus elementos são usados em todos os termos de 4. O procedimento de adaptação dos grânulos depende da posição relativa de $x_{j}$. As condições para expansão do suporte de $M_{j}^{i}$ são:

$$
\begin{aligned}
& \mathrm{SE} \quad \mathrm{x}_{j} \in\left[m p\left(M_{j}^{i}\right)-\frac{\rho}{2}, l_{j}^{i}\right] \text { ENTÃO } \quad l_{j}^{i}(\text { novo })=x_{j}, \\
& \mathrm{SE} \quad \mathrm{x}_{j} \in\left[L_{j}^{i}, m p\left(M_{j}^{i}\right)+\frac{\rho}{2}\right] \text { ENTÃO } \quad L_{j}^{i}(\text { novo })=x_{j},
\end{aligned}
$$

Já os parâmetros do núcleo do conjunto fuzzy trapezoidal são atualizados recursivamente a partir de:

$$
\begin{gathered}
\lambda_{j}^{i}(\text { novo })=\frac{\left(w^{i}-1\right) \lambda_{j}^{i}+x_{j}}{w^{i}}, \\
\Lambda_{j}^{i}(\text { novo })=\frac{\left(w^{i}-1\right) \Lambda_{j}^{i}+x_{j}}{w^{i}} .
\end{gathered}
$$

em que $w^{i}$ representa o número de vezes que o grânulo $\gamma^{i}$ foi escolhido para ser adaptado (Leite et al., 2015). O algoritmo possui ainda um procedimento para remoção de grânulos inativos, e para mescla de grânulos próximos, vide (Leite and Gomide, 2012).

\section{RESULTADOS}

Neste trabalho a aplicação desenvolvida tem como objetivo obter um modelo estável para controlar a navegação de um veículo inteligente em uma via sem intervenções 
externas. Para isso, são utilizados dados do esterçamento e pose do veículo provenientes do ambiente de simulação CARLA (Dosovitskiy et al., 2017) capturados por sensores embarcados. Dessa forma, o modelo controlador deve ser capaz de manter o veículo no centro da via em um caminho determinado. Nesse sentido, o modelo fuzzy do sistema não é previamente conhecido e sua modelagem é determinada pelo fluxo de dados provenientes dos sensores embarcados no veículo no ambiente simulado.

Figura 2. Ilustrações do ambiente de simulação utilizado.
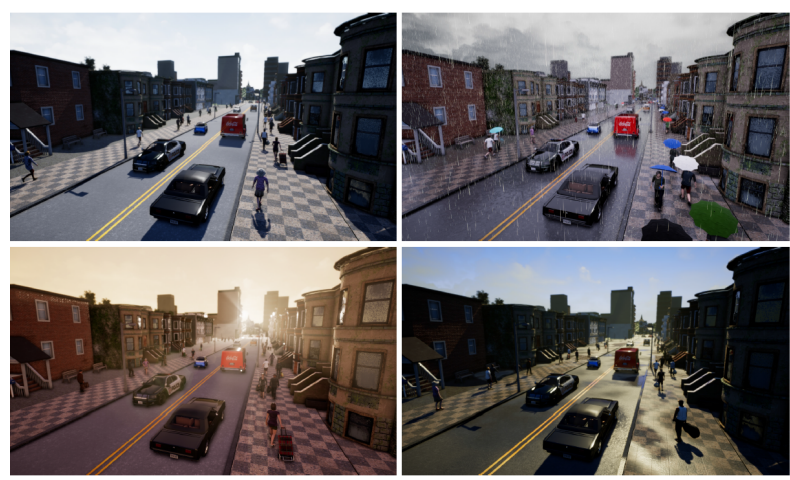

Fonte: Dosovitskiy et al. (2017)

Assim, tenta-se estimar os valores do esterçamento do volante do veículo a partir de dados coletados em sua navegação. O algoritmo é processado 5 vezes e seu resultado é sumarizado na Tabela 1. Nenhuma informação é conhecida antes do processo de aprendizagem iniciar. O resultado dessa predição também pode ser observado na Figura 3.

Figura 3. Estimação do esterçamento obtido com o modelo fuzzy evolutivo e a evolução do conjunto de regras com o fluxo de dados.

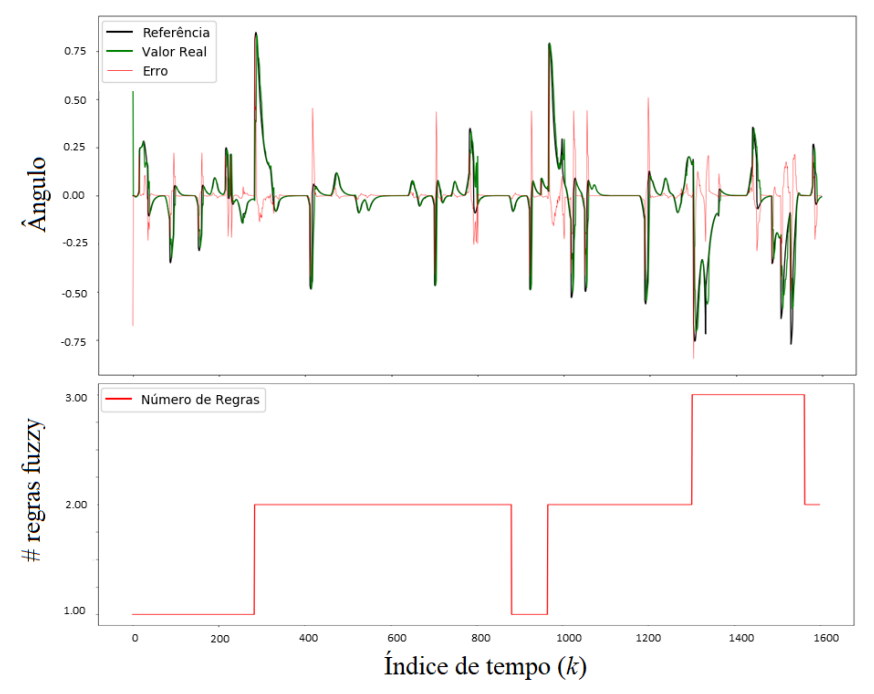

Pode ser visto na Figura 3 a efetividade da abordagem evolutiva na modelagem (para controle) de sistemas nãolineares em que não há conhecimento prévio acerca dos dados e equações. A amplitude do erro é relativamente pequena se comparada às amplitudes dos estados do sistema e, em geral, são visualizadas em mudanças abruptas no sentido do sinal. Neste estudo, os meta-parâmetros do algoritmo de construção do modelo-controlador são $\rho=1,2, h_{r}=1 / \psi=600$ e $\eta=0,5$, vide (Leite, 2012). Estes definem a granularidade máxima, a taxa que controla a quantidade de grânulos, e a taxa de exclusão de grânulos, respectivamente. Neste caso, como o valor de $\rho$ é alto, mostra-se que os grânulos do modelo têm uma região de expansão relativamente grande. Caso $\rho$ fosse menor, os grânulos se expadiriam menos, e mais grânulos seriam necessários para cobrir os dados. O valor de $h_{r}$, por sua vez, indica que se uma tendência não surge ao longo 600 amostras, ela é removida do modelo. Sendo assim, considerando-se a taxa de amostragem do sistema em $10 \mathrm{~Hz}$, pode-se dizer que a cada minuto, em tempo de simulação, caso uma tendência não seja apresentada, ela é removida do modelo. Na Figura 3 também pode ser visualizada a evolução da base de regras fuzzy.

O desempenho do sistema foi avaliado a partir da raiz do erro quadrado médio (RMSE, Root Mean Square Error) das estimações numéricas com relação a saída desejada, provida pelo ambiente CARLA, i.e.,

$$
R M S E=\sqrt{\frac{1}{K} \sum_{k=1}^{K}(y(k)-\bar{y}(k))^{2}},
$$

sendo $K$ o número total, atual, de amostras analisadas; $y$ é o valor real da posição angular no passo $k$; e $\bar{y}$ é o valor estimado pelo modelo fuzzy no passo de tempo $k$.

Tabela 1. Resumo dos resultados para predição da posição angular do volante.

\begin{tabular}{|l|l|c|c|}
\hline $\begin{array}{l}\text { Modelo- } \\
\text { controle }\end{array}$ & $\begin{array}{l}\text { Média de } \\
\text { Regras }\end{array}$ & $\begin{array}{l}\text { Melhor } \\
\text { RMSE }\end{array}$ & $\begin{array}{l}\text { Média } \\
\text { RMSE }\end{array}$ \\
\hline $\begin{array}{l}\text { Fuzzy } \\
\text { Evolutivo }\end{array}$ & $2,27 \pm 0.4$ & $0,0306 \pm 0,0158$ & $0,0368 \pm 0,0142$ \\
\hline ANFIS & $2,15 \pm 0,3$ & $0,2661 \pm 0,0087$ & $0,2674 \pm 0,0018$ \\
\hline
\end{tabular}

De forma comparativa, o desempenho foi confrontado com resultados obtidos com o método de Adaptive-Network based Fuzzy Inference System (ANFIS). Este é um sistema de inferência fuzzy implementado no âmbito de redes neurais adaptativas que une a capacidade de processar variáveis linguísticas de um sistema de inferência à capacidade de adaptação e aprendizagem das redes neurais. Em relação aos resultados mostrados na Tabela 1, a abordagem fuzzy evolutiva é mais precisa a partir do índice $R M S E$, mesmo tendo uma performance pior em relação ao número de regras criadas. Isso decorre principalmente por conta das peculiaridades estruturais e do algoritmo de aprendizado da abordagem fuzzy evolutiva.

A partir do sinal de saída do controlador evolutivo, podese analisar a posição relativa do veículo em relação ao centro da via, como mostrado na imagem da Figura 4. Pode ser visto que o veículo conseguiu se manter próximo à referência em quase todo percurso, desviando-se somente em algumas das amostras, quando da existência de mudanças abruptas na pose do veículo - situações que invariavelmente trazem uma dificuldade adicional ao controlador. 
Figura 4. Avaliação da posição relativa do veículo em relação ao centro da via.

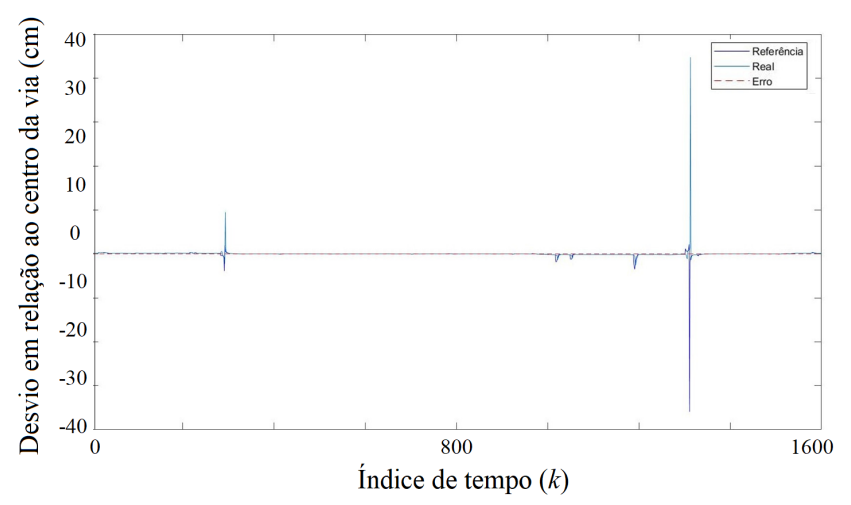

AGRADECIMENTOS

O último autor agradece o apoio do Instituto Serrapilheira, número do processo Serra - 1812-26777, e à Fundação de Amparo à Pesquisa do Estado de Minas Gerais (FAPEMIG), processo APQ-03384-18.

\section{CONCLUSÃO}

Este trabalho teve como objetivo avaliar uma abordagem de modelagem e controle granular fuzzy evolutivo para navegação de um veículo inteligente equipado com sensores embarcados. A abordagem se mostrou capaz de lidar com variações do fluxo de dados provenientes do ambiente de simulação de navegação CARLA. Comparando-se à outra técnica de controle inteligente, ANFIS, que também utiliza conceitos fuzzy, a abordagem evolutiva granular obteve melhor desempenho médio e máximo, tanto na estimação do ângulo de esterçamento, como na manutenção do veículo no centro de uma via. Em trabalhos futuros, esperase implementar o sistema de controle em uma ambiente real, e considerar o controle de outras variáveis, como, por exemplo, a velocidade linear do veículo.

\section{REFERÊNCIAS}

Abhishek, K., Kumar, A., Ranjan, R., and Kumar, S. (2012). A rainfall prediction model using artificial neural network. In Control and System Graduate Research Colloquium (ICSGRC), 2012 IEEE, 82-87. IEEE.

Angelov, P., Filev, D.P., and Kasabov, N. (2010). Evolving intelligent systems: methodology and applications, volume 12. John Wiley \& Sons.

Bargiela, A. and Pedrycz, W. (2008). Toward a theory of granular computing for human-centered information processing. IEEE Trans on Fuzzy Syst, 16(2), 320-330.

Bojarski, M., Testa, D., Dworakowski, D., Firner, B., Flepp, B., Goyal, P., et al. (2016). End to end learning for self-driving cars. arXiv preprint arXiv:1604.07316.

Boyd, S., El Ghaoui, L., Feron, E., and Balakrishnan, V. (1994). Linear matrix inequalities in system and control theory, volume 15. Siam.

Campbell, M., Egerstedt, M., How, J.P., and Murray, R.M. (2010). Autonomous driving in urban environments: approaches, lessons and challenges. Philosophical Transactions of the Royal Society of London A: Mathematical, Physical and Eng Sciences, 368(1928), 4649-4672.
Cara, A.B., Pomares, H., Rojas, I., Lendek, Z., and Babuška, R. (2010). Online self-evolving fuzzy controller with global learning capabilities. Evolving Systems, 1(4), 225-239.

Chen, Z. and Huang, X. (2017). End-to-end learning for lane keeping of self-driving cars. In Intelligent Vehicles Symposium (IV), 2017 IEEE, 1856-1860. IEEE.

Codevilla, F., Müller, M., Dosovitskiy, A., López, A., and Koltun, V. (2017). End-to-end driving via conditional imitation learning. arXiv preprint arXiv:1710.02410.

Dosovitskiy, A., Ros, G., Codevilla, F., Lopez, A., and Koltun, V. (2017). Carla: An open urban driving simulator. arXiv preprint arXiv:1711.03938.

Feng, G. (2002). An approach to adaptive control of fuzzy dynamic systems. IEEE Transactions on Fuzzy Systems, $10(2), 268-275$.

Kasabov, N., Zhou, L., Doborjeh, M., Doborjeh, Z., and Yang, J. (2017). New algorithms for encoding, learning and classification of fmri data in a spiking neural network architecture: A case on modeling and understanding of dynamic cognitive processes. IEEE Trans on Cognitive and Developmental Syst, 9(4), 293-303.

Landau, I.D., Lozano, R., M'Saad, M., and Karimi, A. (2011). Adaptive control: algorithms, analysis and applications. Springer Science \& Business Media.

Leite, D. (2012). Evolving Granular Systems. Ph.D. thesis, University of Campinas, School of Electrical and Computer Engineering.

Leite, D. and Gomide, F. (2012). Evolving linguistic fuzzy models from data streams. In Trillas E., Bonissone P., Magdalena L., Kacprzyk J. (eds) Combining Experimentation and Theory. Studies in Fuzziness and Soft Computing, 271, 209-223. Springer: Berlin, Heidelberg.

Leite, D., Palhares, R.M., Campos, V.C.S., and Gomide, F. (2015). Evolving granular fuzzy model-based control of nonlinear dynamic systems. IEEE Transactions on Fuzzy Systems, 23(4), 923-938.

Leite, D., Ballini, R., Costa, P., and Gomide, F. (2012). Evolving fuzzy granular modeling from nonstationary fuzzy data streams. Evolving Systems, 3(2), 65-79.

Majdani, F., Petrovski, A., and Doolan, D. (2018). Evolving ann-based sensors for a context-aware cyber physical system of an offshore gas turbine. Evolving Systems, $9(2), 119-133$.

SAE et al. (2016). Taxonomy and definitions for terms related to on-road motor vehicle automated driving systems. SAE Standard J3016, 01-16.

Sauer, A., Savinov, N., and Geiger, A. (2018). Conditional affordance learning for driving in urban environments. arXiv preprint arXiv:1806.06498.

Singh, S. (2015). Critical reasons for crashes investigated in the national motor vehicle crash causation survey. Technical report.

Škrjanc, I., Iglesias, J., Sanchis, A., Leite, D., Lughofer, E., and Gomide, F. (2019). Evolving fuzzy and neuro-fuzzy approaches in clustering, regression, identification, and classification: A survey. Info Sci, 490(2), 344-368.

Yao, Y. (2010). Human-inspired granular computing. Novel developments in granular computing: applications for advanced human reasoning and soft comput., 1-15. 